\title{
Three-dimensional wave scattering by a fixed cylindrical inclusion submerged in a fluid medium
}

\author{
A.J.B. Tadeu* ${ }^{*}$ L.M.C. Godinho \\ Department of Civil Engineering, University of Coimbra, 3049, Coimbra, Portugal
}

Received 24 February 1999; accepted 28 June 1999

\begin{abstract}
This paper presents the solution for a fixed cylindrical irregular cavity of infinite length submerged in a homogeneous fluid medium, and subjected to dilatational point sources placed at some point in the fluid. The solution is first computed for a wide range of frequencies and wavenumbers, which are then used to obtain time-series by means of (fast) inverse Fourier transforms into space-time.

The method and the expressions presented are implemented and validated by applying them to a fixed cylindrical circular cavity submerged in an infinite homogeneous fluid medium subjected to a point pressure source for which the solution is calculated in closed form.

The boundary elements method is then used to evaluate the wave-field elicited by a point pressure source in the presence of fixed rigid cylindrical cavities, with different cross-sections, submerged in an unbounded fluid medium and in a half-space. Simulation analyses with this idealized model are then used to study the patterns of wave propagation in the vicinity of these inclusions. The amplitude of the wavefield in the frequency vs axial-wavenumber domain is presented, allowing the recognition, identification, and physical interpretation of the variation of the wavefield. (C) 1999 Elsevier Science Ltd. All rights reserved.
\end{abstract}

Keywords: Wave propagation; Submerged fixed cylindrical cavity; Point pressure source; Scattering; Boundary element method; Two-and-a-half-dimensional problem

\section{Introduction}

The behaviour of waves as they propagate in a semi-infinite medium with discontinuities has drawn the attention of many researchers over the years. Some of the first analytical studies on wave diffraction and scattering were concerned with the problem of wave motion and reverberations in alluvial basins of regular shape [1,2], and with the issue of wave scattering induced by cavities [3-6]. More recently, semi-analytical methods have been used to analyse wave diffraction caused by geological irregularities of arbitrary shape within globally homogeneous media [7-9]. By contrast, the application of purely numerical methods (i.e. finite elements or differences combined with boundaries) has been restricted, for the most part, to situations where the response is required only within localized irregular domains, such as soil-structure interaction problems [1012]. Discrete methods have also occasionally been used to model large alluvial basins, under plane-strain conditions [13]. A boundary integral equation method has been applied [14] to compute the two-dimensional (2D) acoustic scattering field arising from deformations of an oceanic

\footnotetext{
*Corresponding author. Tel.: +351-039-410675; fax: +351-039-410111.
}

waveguide's surfaces. This same method was used $[15,16]$ to obtain the 2D acoustic scattered field generated by objects embedded between two half-spaces with different densities. More recently, a boundary integral formulation for the analysis of acoustic barriers over an impedance plane as infinitely thin structures has been presented [17].

Hybrid methods involving a combination of finite elements to model the interior domain containing the inhomogeneities and semi-analytical representations for the exterior domain have been used [18]. A transitional matrix solution for the spectral scattering response of a partially buried 3D elastic obstacle in a plane stratified fluid media has been formulated [19].

The application of most of these numerical methods has been restricted to situations where the solution is required within 2D domains. The evaluation of the full scattering wavefield generated by sources placed in the presence of $3 \mathrm{D}$ propagation media requires the use of computationally demanding numerical schemes.

The solution becomes much simpler if the medium is $2 \mathrm{D}$, even if the dynamic source remains 3D, a point load, for example. Such a situation is frequently referred to as a twoand-a-half-dimensional problem (or 2-1/2-D for short), for which solutions can be obtained by means of a two spatial 


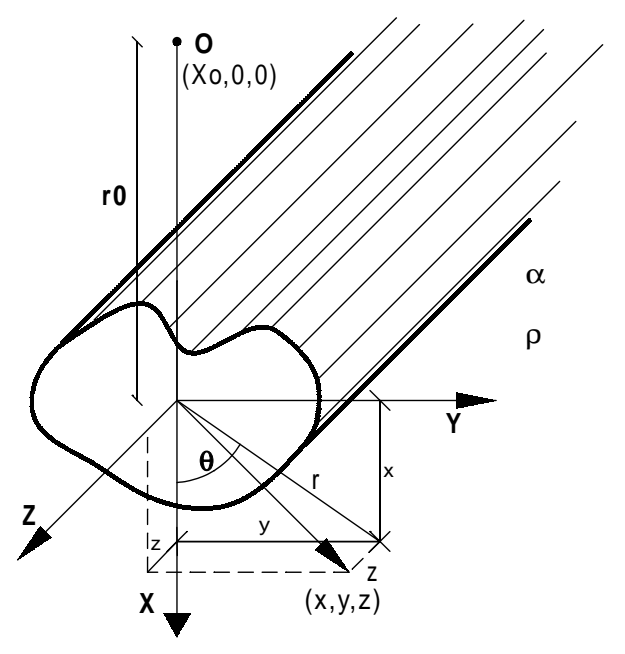

Fig. 1. Geometry of the problem.

Fourier transform in the direction in which the geometry does not vary. This requires solving a sequence of $2 \mathrm{D}$ problems with different spatial wavenumbers $k_{z}$. Then, using the inverse Fourier transform, the 3D field can be synthesized.

This solution is known in closed form for inclusions with simple geometry, such as a circular cylinder, for which the wave equation is separable. However if the inclusion has an irregular cross-section the solution is more difficult to obtain. This paper presents the solution obtained for a fixed cylindrical cavity of infinite length submerged in a homogeneous fluid medium, and subjected to dilatational point sources placed at some point in the fluid, using boundary elements.

The solution at each frequency is expressed in terms of waves with varying wavenumber $k_{z}$, (with $z$ being the direction in which the geometry does not vary), which is subsequently Fourier transformed into the spatial domain. The wavenumber transform in discrete form is obtained by considering an infinite number of virtual point sources equally spaced along the $z$-axis and at a sufficient distance from each other to avoid spatial contamination [20]. In addition, the analyses are performed using complex frequencies, shifting down the frequency axis, in the complex plane, in order to remove the singularities on (or near) the axis, and to minimize the influence of the neighbouring fictitious sources [21].

The method presented is implemented and validated by applying it to a fixed cylindrical circular cavity submerged in an infinite homogenous fluid medium subjected to a point pressure source for which the solution is calculated in closed form.

The boundary elements method is then used to evaluate the wavefield elicited by a point pressure source in the presence of a fixed rigid cylindrical cavity submerged in an unbounded fluid medium and in a half-space. Simulation analyses with this idealized model are used to study the patterns of wave propagation in the vicinity of these inclusions when different cylindrical cross-sections with a common perimeter are used. The amplitude of the wavefield in the frequency vs axial-wavenumber domain is presented, allowing the recognition, identification, and physical interpretation of the variation of the wavefield.

\section{Problem statement}

Consider a cylindrical irregular inclusion of infinite extent, submerged in a spatially uniform fluid medium (Fig. 1), subjected to a harmonic point pressure source at position $\left(x_{0}, 0,0\right)$, oscillating with a frequency $\omega$.

$p_{\text {inc }}=\frac{A \mathrm{e}^{\mathrm{i}(\omega / \alpha)\left(\alpha t-\sqrt{\left.\left(x-x_{0}\right)^{2}+y^{2}+z^{2}\right)}\right.}}{\sqrt{\left(x-x_{0}\right)^{2}+y^{2}+z^{2}}}$

in which the subscript inc denotes the incident field, $A$ is the wave amplitude, $\alpha$ is the pressure wave velocity of the medium, and $\mathrm{i}=\sqrt{-1}$.

Defining the effective wavenumbers

$k_{\alpha}=\sqrt{\frac{\omega^{2}}{\alpha^{2}}-k_{z}^{2}}, \quad \operatorname{Im} k_{\alpha}<0$

by means of the axial wavenumber $k_{z}$, and Fourier-transforming Eq. (1) in the $z$ direction, one obtains

$\hat{p}_{\text {inc }}\left(\omega, x, y, k_{z}\right)=\frac{-\mathrm{i} A}{2} H_{0}^{(2)}\left(k_{\alpha} \sqrt{\left(x-x_{0}\right)^{2}+y^{2}}\right)$

in which the $H_{n}^{(2)}(\cdots)$ are second Hankel functions of order $n$.

If one considers an infinite set of periodically placed sources along the $z$ direction at equal intervals, $L$, the incident field may be written as

$p_{\text {inc }}(\omega, x, y, z)=\frac{2 \pi}{L} \sum_{m=-\infty}^{\infty} \hat{p}_{\text {inc }}\left(\omega, x, y, k_{z}\right) \mathrm{e}^{-\mathrm{i} k_{z m} z}$

with $k_{z m}=(2 \pi / L) m$. This equation converges and can be approximated by a finite sum of terms.

\section{Boundary element formulation}

The boundary element method (BEM) is used to obtain the 3D field generated by a cylindrical inclusion with an irregular shape. In the case of an acoustic medium, the 21/2-D problem can be solved as a discrete summation of $2 \mathrm{D}$ BEM for different $k_{z}$ wavenumbers. Then, using the inverse Fourier transform, the 3D field can be synthesized. The wavenumber transform in discrete form is obtained, as explained above, by considering an infinite number of virtual point sources equally spaced along the $z$-axis and at a sufficient distance from each other to avoid spatial contamination [20].

Considering the literature currently available on the $\mathrm{BEM}$, it does not appear relevant to repeat yet again the details of the formulation required for the type of the scattering problem presented herein (see for example Ref. [22]). 


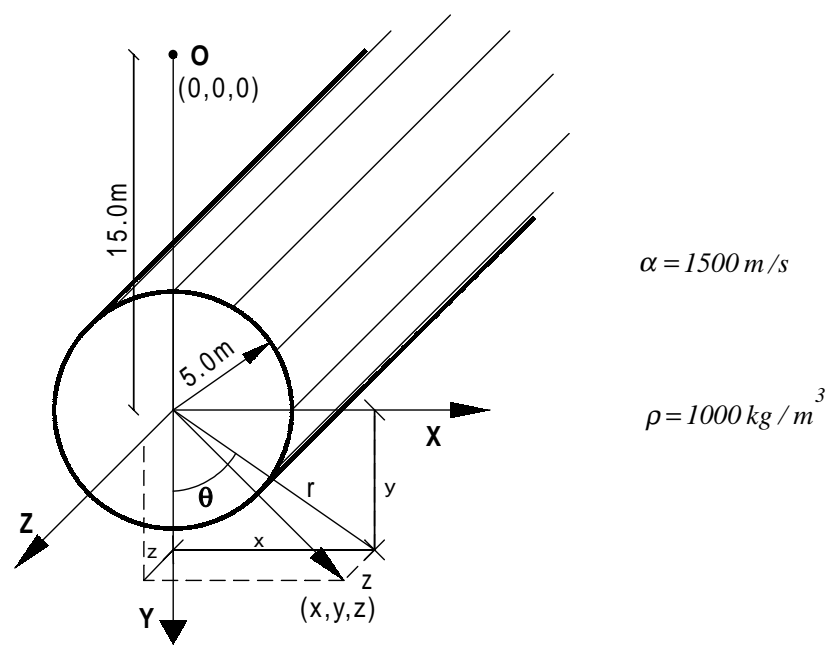

Fig. 2. Circular cylindrical inclusion in an unbounded medium.

It suffices to state that each 2D BEM solution requires the evaluation of the integral

$H^{k l}=\int_{C_{l}} H\left(\underline{x}_{k}, \underline{x}_{l}, n_{l}\right) \mathrm{d} C_{l}$

in which $H^{k l}$ is the pressure velocity component at $x_{k}$ due to pressure load at $\underline{x}_{l}$, and $n_{l}$ is the unit outward normal for the $l$ th boundary segment $C_{l}$. In the present case, the required pressure velocity function is

$H\left(\underline{x}_{k}, \underline{x}_{l}, n_{l}\right)=\frac{-\mathrm{i} k_{\alpha}}{4 \rho} H_{l}^{(2)}\left(k_{\alpha} r\right) \frac{\partial r}{\partial n_{l}}$.

In this equation $\rho$ is the mass density, $r$ is the source-receiver distance on the plane $x y$, and $\mathrm{i}=\sqrt{-1}$.

Mathematical manipulation of the integral equations, combined and subjected to null normal particle velocity at the inclusion boundary, and discretized appropriately, gives a system of equations that can be solved for the nodal pressures. The required integrations in Eq. (5) are performed by means of Gauss-Legendre quadrature, using four integration points.

The scattered pressure field in the fluid is then defined as a function of the nodal pressure values, as follows:

$p_{\text {sca }}^{k}=\sum_{l=1}^{N} p_{l} H^{k l}$

where $p_{\text {sca }}^{k}$ is the scattered pressure field at receiver $k, N$ is the total number of boundary elements and $p^{l}$ is the nodal pressure value at element $l$.

\section{Pressure in time-space}

The pressures in the spatial-temporal domain are obtained by a numerical fast Fourier transform in $k_{z}$, considering a source whose temporal variation is given by a Ricker wavelet, as defined below. This wavelet form is chosen because it decays rapidly, in both time and frequency; this not only reduces the computational effort, but allows easier interpretation of the computed time-series and synthetic waveforms. Notice that the type of wavelet only defines the frequency domain where the BEM solution is required. Shorter duration pulses are better identified in the time domain, but they require a larger number of frequency responses to be calculated.

The Ricker wavelet function is given by

$u(\tau)=A\left(1-2 \tau^{2}\right) \mathrm{e}^{-\tau^{2}}$

where $A$ is the amplitude, $T=\left(t-t_{\mathrm{s}}\right) / t_{0}$ and $t$ denotes time; $t_{\mathrm{s}}$ is the time when the maximum occurs, while $\pi t_{0}$ is the characteristic (dominant) period of the wavelet. Its Fourier transform is

$U(\omega)=A\left[2 \sqrt{\pi t_{0}} \mathrm{e}^{-\mathrm{i} \omega t_{s}}\right] \Omega^{2} \mathrm{e}^{-\Omega^{2}}$

in which $\Omega=\omega t_{0} / 2$.

As stated before, the Fourier transformations are achieved by discrete summations over wavenumbers and frequencies, which is mathematically equivalent to adding periodic sources at spatial intervals $L=2 \pi / \Delta k_{z}$ (in the $z$ axis), and temporal intervals $T=2 \pi / \Delta \omega$, with $\Delta k_{z}$ and $\Delta \omega$ being the wavenumber and frequency steps, respectively [1]. The spatial separation $L$ must be sufficiently large to avoid contamination of the response by the periodic sources. In other words, the contribution to the response by the fictitious sources must be guaranteed to occur at times later than $T$. This goal can also be aided substantially by shifting the frequency axis slightly downward, that is, by considering complex frequencies with a small imaginary part of the form $\omega_{\mathrm{c}}=\omega-\mathrm{i} \eta$ (with $\eta=0.7 \Delta \omega$ ). This technique results in a significant attenuation or virtual elimination of the periodic sources. In the time domain, this shift is later taken into account by applying an exponential window $\mathrm{e}^{\eta t}$ to the response [23]. 

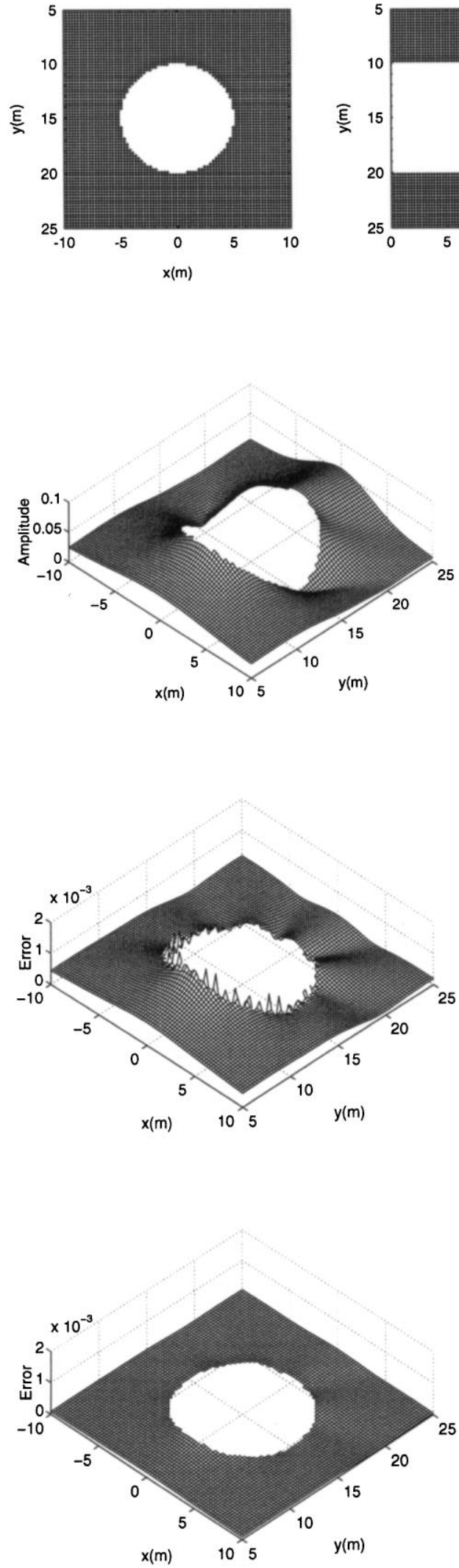

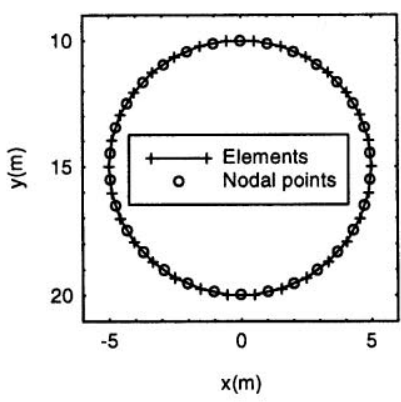

Typical BEM mesh and receivers grid.

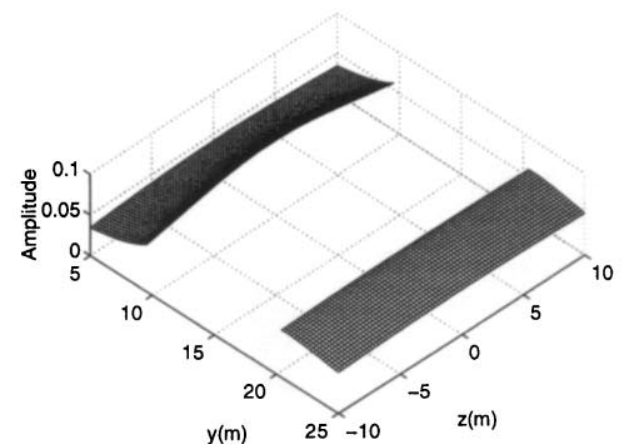

Analytical solution

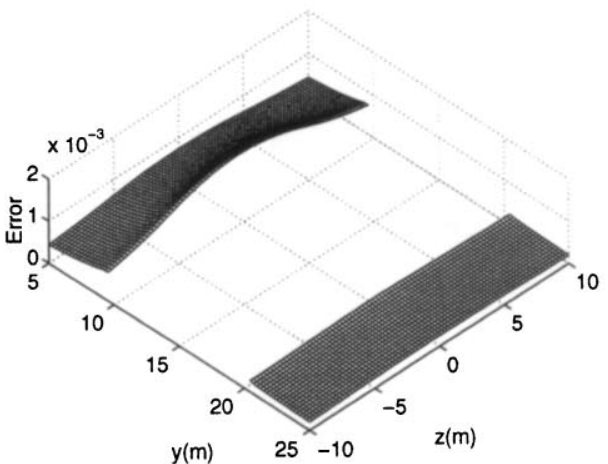

Error using 42 boundary elements.

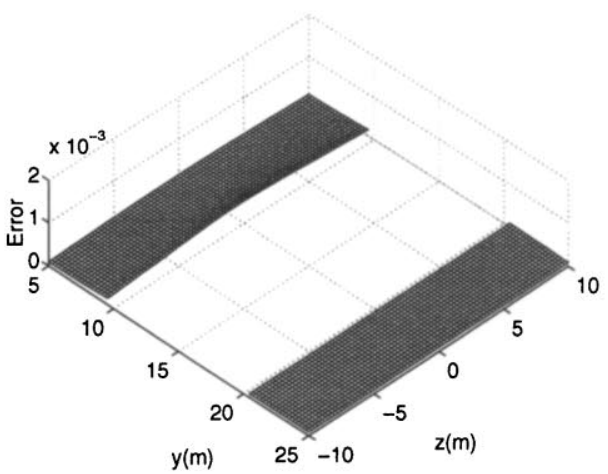

Error using 84 boundary elements.

Fig. 3. Scattered pressure field. Harmonic pressure load of $200 \mathrm{~Hz}$. 

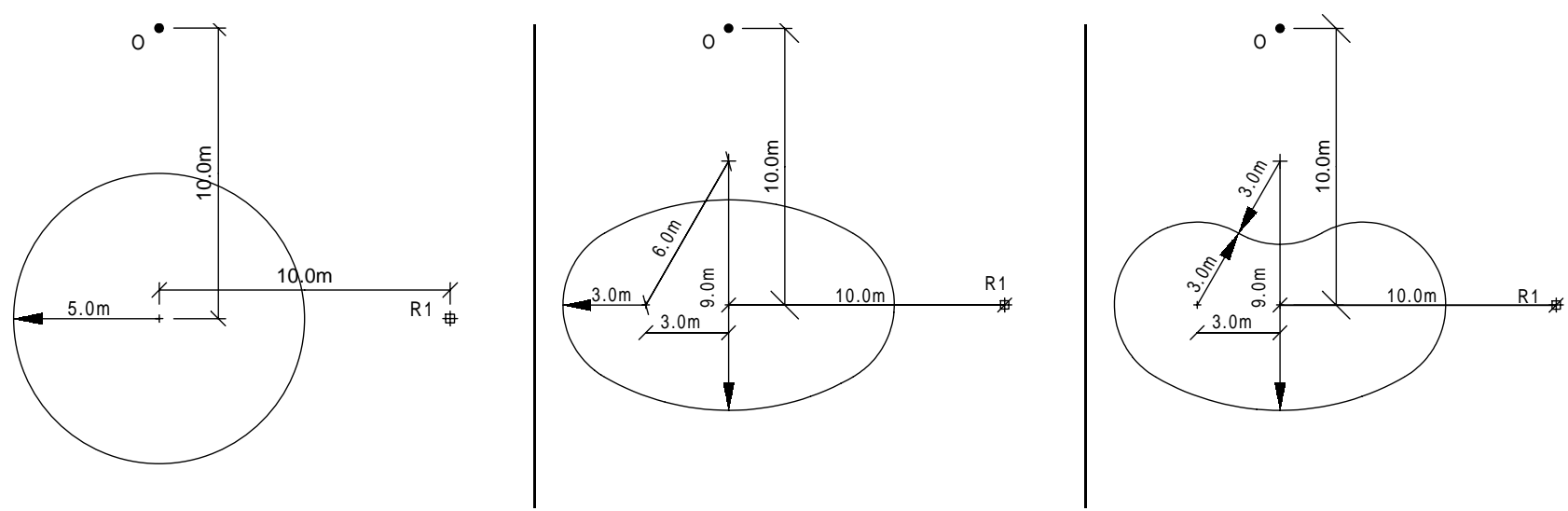

Fig. 4. Cross-section of the fixed cylindrical irregular cavities.

\section{Validation of the BEM algorithm}

The BEM algorithm was implemented and validated by applying it to a fixed cylindrical circular cavity, submerged in a homogeneous fluid medium, subjected to a harmonic point pressure load applied at point $\mathrm{O}$, as in Fig. 2, for which the solution is known in closed form and described in Appendix A.

The response is calculated over a fine vertical grid plane, placed along the longitudinal axis and perpendicular to it as illustrated in Fig. 3. This figure also displays an example of a BEM mesh, including the nodal points in the middle of each constant boundary element. Fig. 3 represents the scattered pressure field computed when a harmonic pressure load of $200 \mathrm{~Hz}$ is excited. In these figures, both the response obtained with the closed form solution and the amplitude of the difference in the response obtained using the BEM (labelled error) when the inclusion is modelled with a different number of boundary elements are displayed. In this case, the Green's function that applies is given by

$G_{\text {full }}\left(\omega, x, y, k_{z}\right)=\frac{\mathrm{i}}{4} H_{0}^{(2)}\left(k_{\alpha} \sqrt{\left(x-x_{\mathrm{p}}\right)^{2}+\left(y-y_{\mathrm{p}}\right)^{2}}\right)$

where $x$ identifies the receiver and $x_{\mathrm{p}}$ gives the position of the concentrated pressure load.

As expected, the BEM accuracy improves as shorter boundary elements are used to model the response. In the present example, the ratio between the wavelength of incident waves to length of boundary elements varies from 10.0 (42 boundary elements) to 20.0 (84 boundary elements). Analysis of the results also makes it possible to verify the improvement of the BEM solution as the distance to the surface of the inclusion increases. It appears to be advantageous to use a higher number of boundary elements when the response is required at points close to the boundary of the cavity, where the response changes rapidly.

\section{Applications}

\subsection{Fixed cylindrical irregular cavities submerged in unbounded fluid medium}

Next we consider the scattered pressure field of fixed rigid cylindrical cavities submerged in an unbounded fluid medium, with different cross-sections: circular, oval and kidney-shaped. The perimeter of all these cavities is kept constant $(10 \pi \mathrm{m})$ as one may conclude from analysing Fig. 4 , which displays the geometry of the cross-section of the inclusions considered herein. At time $t=0$, a point source at a point $\mathrm{O}$ creates a spherical pressure pulse propagating away from $\mathrm{O}$. The field generated is computed at receivers $\mathrm{R} 1$ located in five planes equally spaced $(10 \mathrm{~m})$ along the $z$ direction. The geometry of the plane containing the point source is illustrated in Fig. 4.

The pressure wave velocity $(\alpha=1500 \mathrm{~m} / \mathrm{s})$ and density $\left(\rho=1000 \mathrm{~kg} / \mathrm{m}^{3}\right)$ of the host fluid are kept constant in all the analyses. The computations are performed in the frequency range $2.5-320 \mathrm{~Hz}$, with a frequency increment of $2.5 \mathrm{~Hz}$, which determines the total time duration $(T=$ $0.4 \mathrm{~s}$ ) for the analysis in the time domain. The spatial period considered in the analysis is $L=2 T \alpha=1200 \mathrm{~m}$. The source time dependence is a Ricker wavelet with a characteristic frequency of $128 \mathrm{~Hz}$.

The cavity is modelled with a number of boundary elements that changes with the frequency of excitation of the harmonic load. A ratio between the wavelength of the incident waves to length of the boundary elements is kept at a minimum of 10.0. In any case the minimum number of the boundary elements used is less than 30. Fig. 5 displays the amplitude of the scattered wave field of these responses in the frequency vs axialwavenumber domain, and the correspondent synthetic waveforms $(0-100 \mathrm{~ms})$ of the scattered wave fields obtained in the presence of the different fixed cavities, at the aforementioned receiver. Each plot is normalized with respect to its own maximum. Values of $k_{z}$ in excess of $\omega / \alpha$ correspond to inhomogeneous, evanescent waves which decay rapidly in space. 

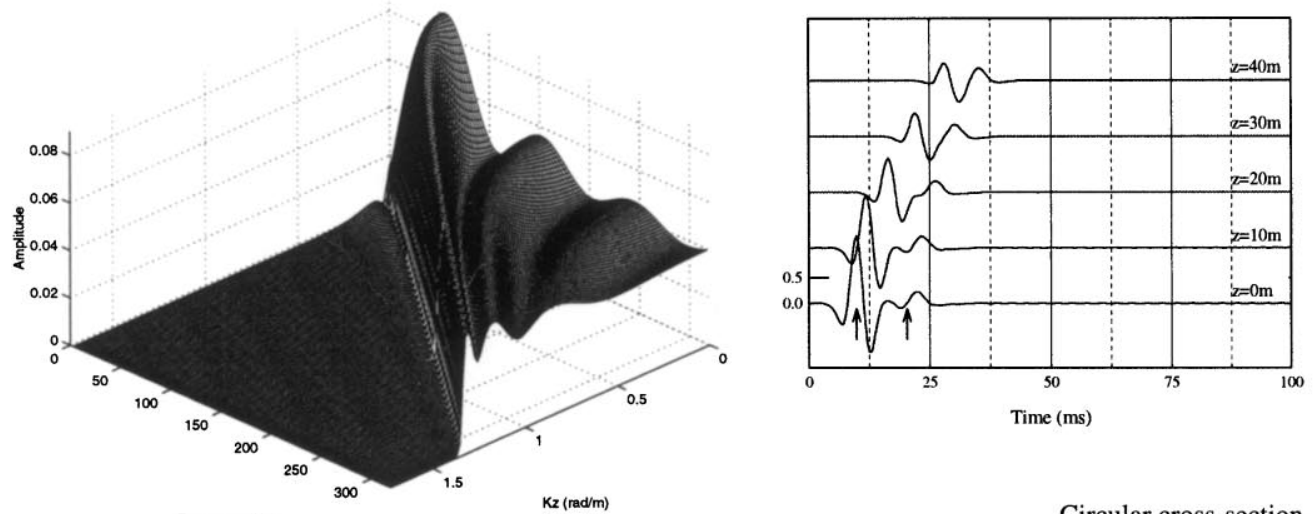

Circular cross-section.
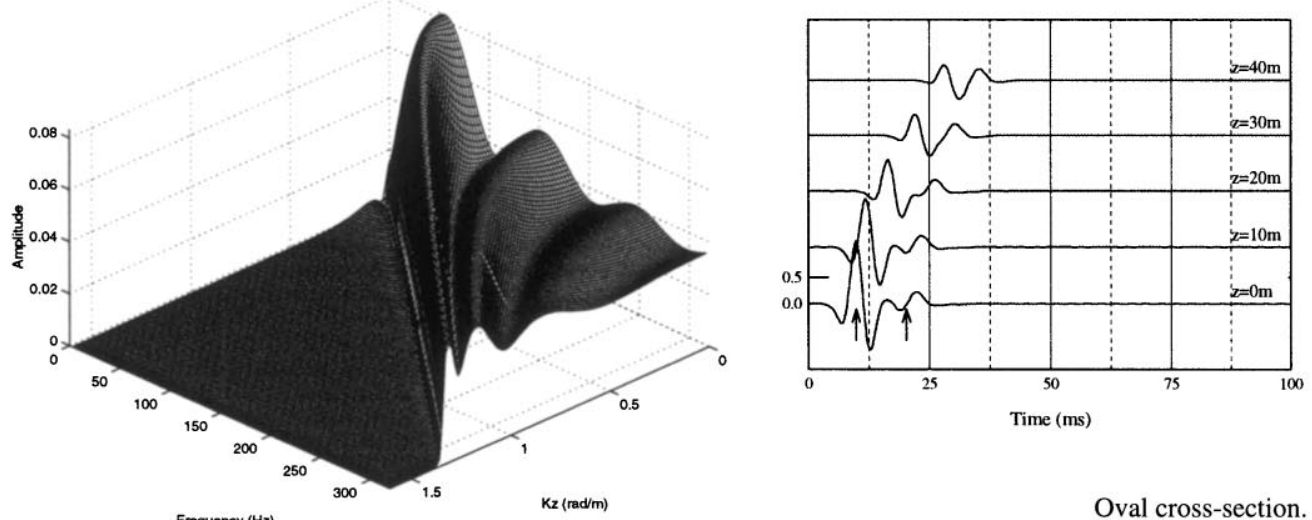

Oval cross-section.
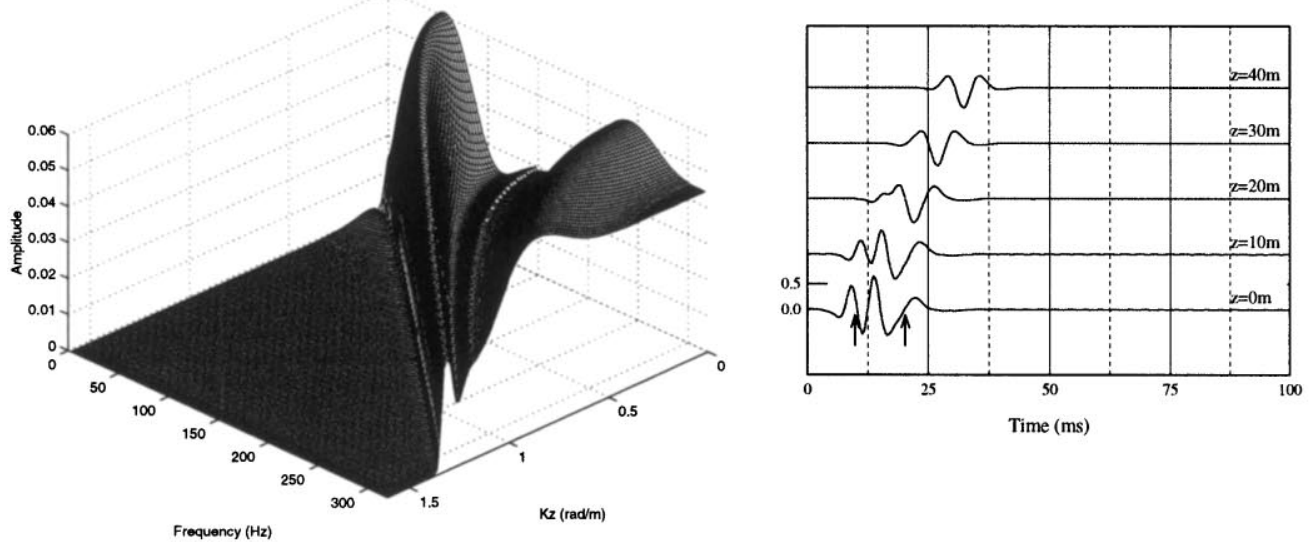

Kidney cross-section.

Fig. 5. Unbounded fluid medium. Responses at receivers R1.

The time records are a sequence of a reflected (scattered) pulse and another pulse that travels around the cylinder (diffracted). A geometric acoustic ray analysis shows that these signals result from the propagation of a reflected and diffracted wavefront on the surface of the inclusion. Associated with each wavefront is a ray; thus there are two principal rays backscattered from such an inclusion. Fig. 6 shows the propagation path of these waves between the source and the receiver located at the plane source.

The travel times of the aforementioned rays, are equal to the corresponding arrival times of the signals in Fig. 6 . Arrows are added in this figure to indicate their arrival. In 


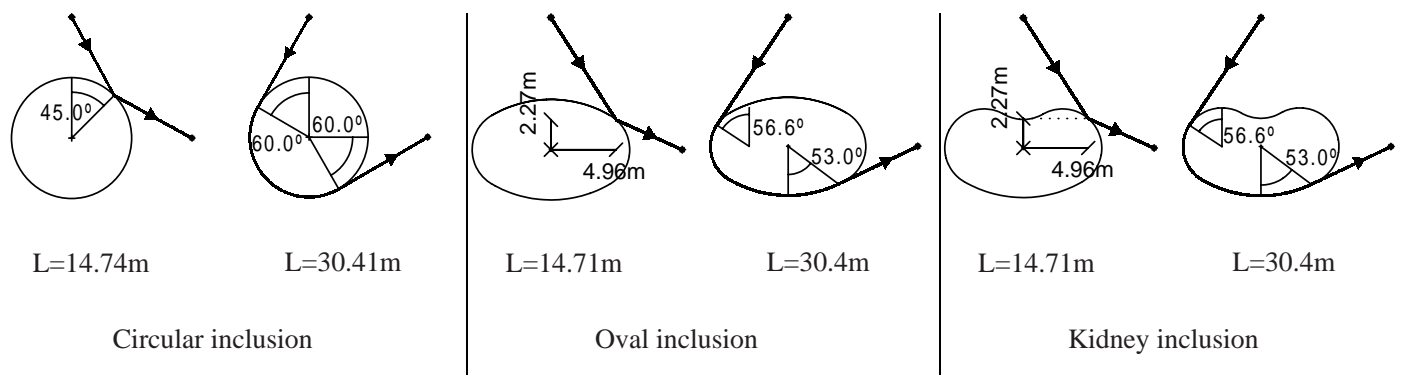

Fig. 6. Propagation path of reflected and diffracted waves travelling between the source $\mathrm{O}$ and the receiver located at the plane source.

the case of the kidney-shaped inclusion, the results reveal complicated wave patterns which originate from reverberations within the concave section of the cavity. Hence, the time signals are more difficult to analyse due to the interference between these different pulses. A ray analysis of the concave part can be quite cumbersome.

The Fourier spectra of the scattered field exhibit pronounced maxima and minima, occurring at particular frequencies and in definite frequency intervals. The periodicity of these peaks and troughs can be understood in terms of the interference of reflected and diffracted waves. The waves interfere constructively when their phase difference is a multiple of $2 \pi$. Thus, for $k_{z}=0$, the frequency interval between successive maxima and minima is $\Delta f=\alpha / \Delta s$, where $\Delta s$ is the travel path difference between the reflected and diffracted pulses $(\Delta f \approx 95.6 \mathrm{~Hz}$ ), which coincides with the results displayed in Fig. 5. Again, the kidney response is influenced by the existence of the reverberations within the concave section of the inclusion. It originates a poorly developed second fold and a more pronounced third fold in the frequency vs wavenumber spectra.

Additionally, the spectra plots show a smoother response for higher frequencies. This was expected, since for higher frequencies the amplitude of the waves travelling around the inclusion decreases, giving place to a shadow zone. This indicates that, in order to observe the interference, the source pulse should be rich in low frequencies.

As $k_{z}$ increases, the frequency distance between peaks and valleys decreases. This fact can be explained because as the inclination of the wave path decreases in relation to the axis of the cylinder (larger $k_{z}$ ) $\Delta s$ increases, implying a smaller distance $\Delta f$.

\subsection{Fixed cylindrical irregular cavities submerged in a half-space}

Consider the inclusions previously analysed submerged in a fluid, bounded by a fixed flat surface (half-space), illuminated by a point source placed at the surface (Fig. 7). The source generates a Ricker wavelet pressure pulse with a centre frequency of $128 \mathrm{~Hz}$. Calculated frequencies range from 2.5 to $320 \mathrm{~Hz}$ with an increment of $2.5 \mathrm{~Hz}$. The response is computed at two receivers placed on the surface.

The BEM uses Green's function for the elastic half-space, expressed as the sum of the pressure fields generated by the real load and a virtual load placed at its mirror position, so as to obtain the suitable boundary conditions at the flat rigid surface

$$
\begin{aligned}
G_{\text {half }}\left(\omega, x, y, k_{z}\right)= & \frac{\mathrm{i}}{4}\left[H_{0}^{(2)}\left(k_{\alpha} \sqrt{\left(x-x_{\mathrm{p}}\right)^{2}+\left(y-y_{\mathrm{p}}\right)^{2}}\right)\right. \\
& \left.+H_{0}^{(2)}\left(k_{\alpha} \sqrt{\left(x-x_{\mathrm{p}}\right)^{2}+\left(y+y_{\mathrm{p}}\right)^{2}}\right)\right]
\end{aligned}
$$

in which $G_{\text {half }}\left(\omega, x, y, k_{z}\right)$ is Green's function for pressure at $x$ due to a concentrated pressure load at $x_{\mathrm{p}}$.

The definition of the number of boundary elements and
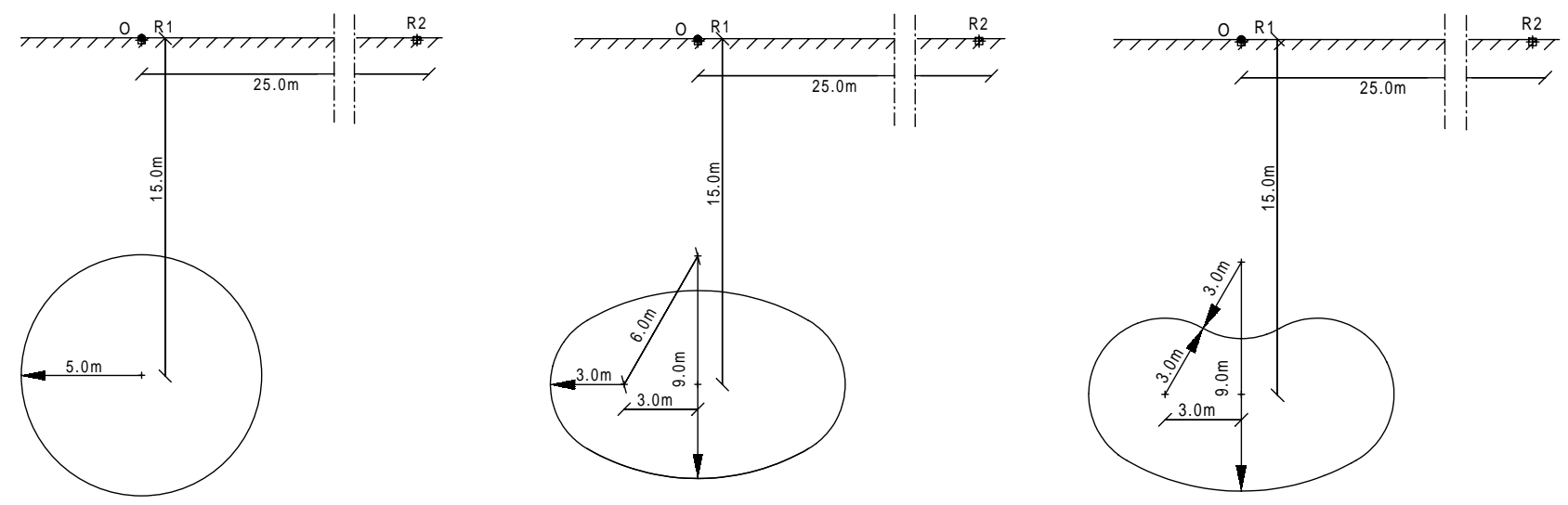

Fig. 7. Cross-section of the fixed cylindrical irregular cavities. 

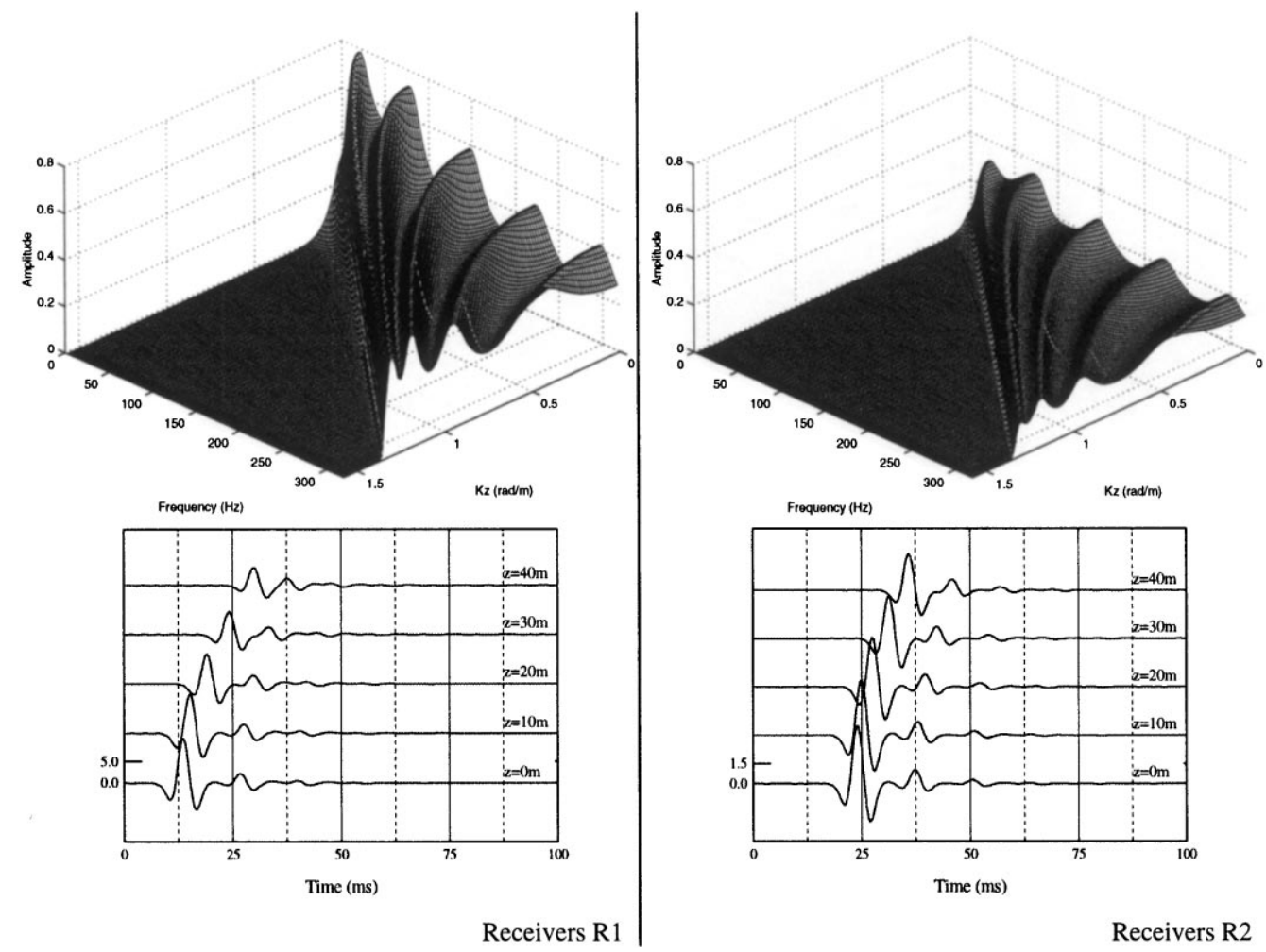

Fig. 8. Half fluid medium. Responses at receivers R1 and R2; circular cross-section.
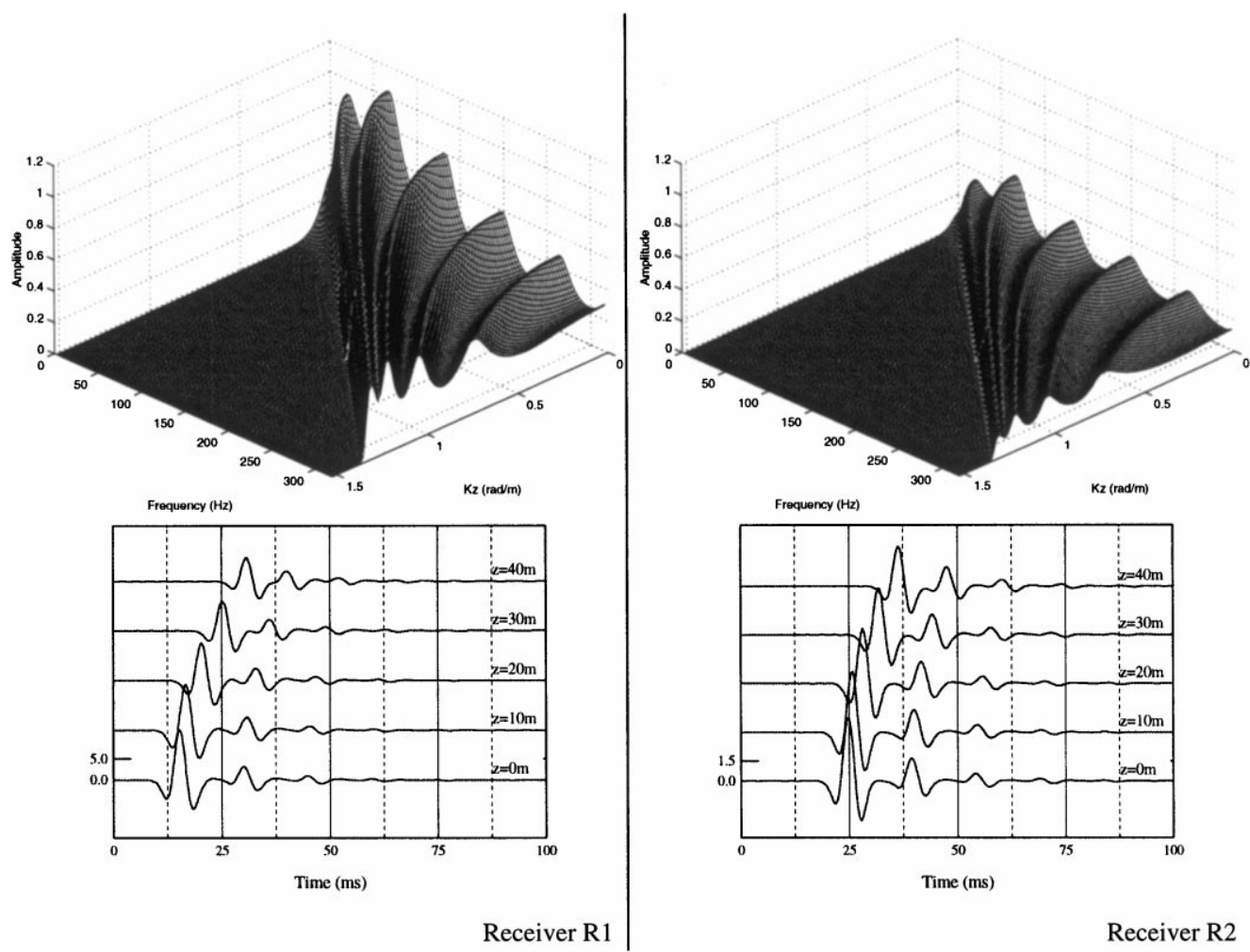

Fig. 9. Half fluid medium. Responses at receivers R1 and R2; oval cross-section. 

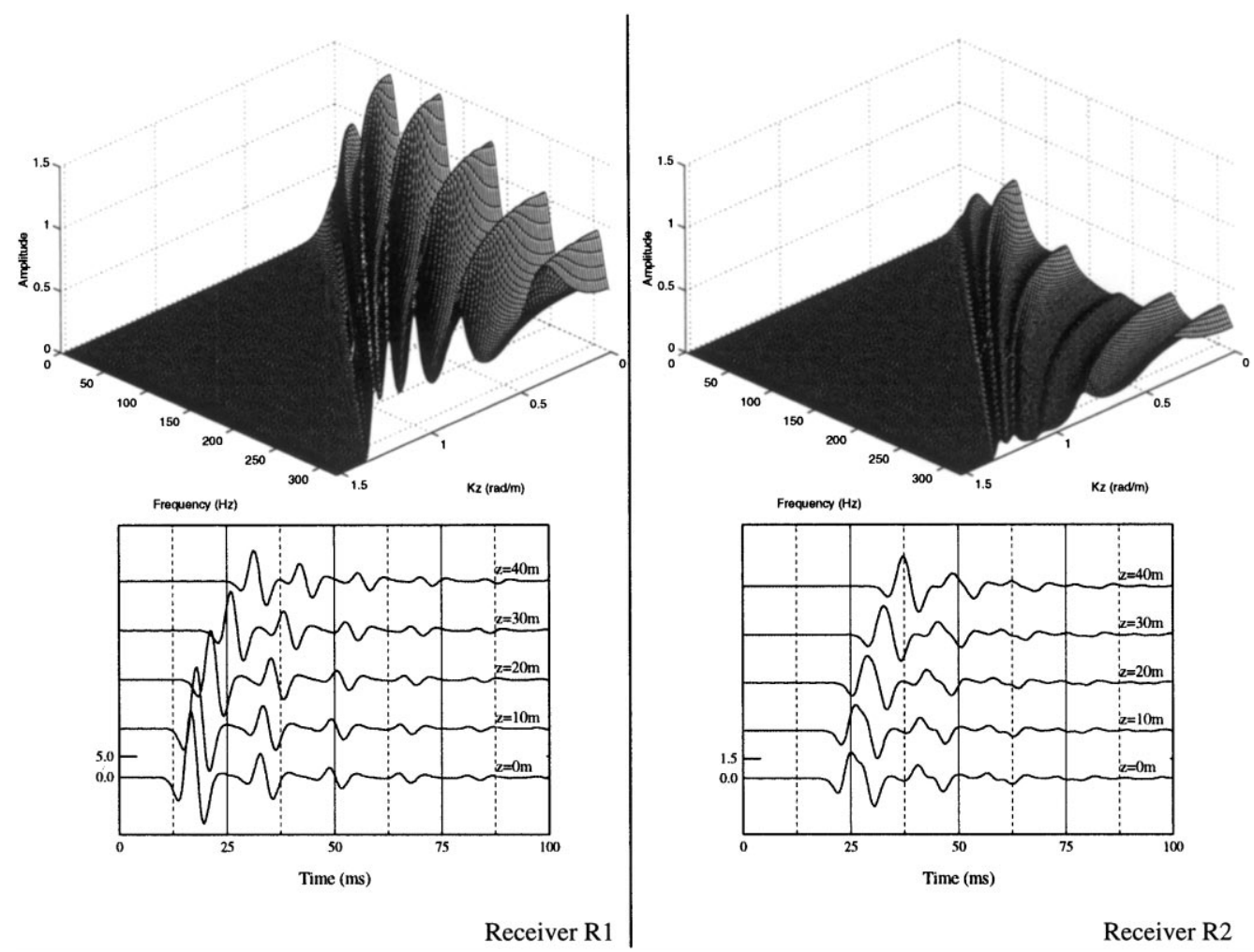

Fig. 10. Half fluid medium. Responses at receivers R1 and R2; kidney cross-section.

the distance between virtual point sources along the $z$ axis follow the same procedures as for the previously applications. The minimum number of the boundary elements used to model the inclusion is again set at 30 .

Figs. 8-10 display the time responses and spectra representations of the scattered field at the receivers. The Fourier amplitude spectra are observed to exhibit pronounced maxima and minima, which occur at particular frequencies and in definite frequency intervals. Both receivers experience similar responses. The periodicity of these peaks and valleys is mainly the result of the interference between waves that are reflected at the top of the inclusion and the flat surface. The frequency intervals between successive maxima and minima are $\Delta f=\alpha / 2 h$, where $h$ is the depth of the inclusion. In the present examples $\Delta f$ (circular) $=$ $75 \mathrm{~Hz}, \Delta f($ oval $)=67 \mathrm{~Hz}$ and $\Delta f$ (kidney $)=62.5 \mathrm{~Hz}$. One may observe that in the case of the kidney-shaped inclusion, the distance $\Delta f$ is not affected by the concave section of the inclusion. Indeed, the depth $h$ is related to the shortest distance from the inclusion to the flat surface. However, the value of amplitude of the spectra response shows evidence of the result of multi-reverberations which originate within the concave section of the cavity.

Notice that there is an additional peak at low frequencies, which is the result of waves diffracted around the cavity. In the absence of these waves the first peak would occur at $f=$ $\alpha / 2 h$. The influence of these waves travelling around the inclusion decreases rapidly as the frequency of excitation increases, as explained in the previous application. Thus, the position of the folds that follow the first fold is less and less affected as the frequency increases.

The time signals at each receiver are a sequence of pulses resulting from the reflection and diffraction of waves at the boundary of the cavity and at the fixed flat surface. The first pulse arrival corresponds to pulses that are directly reflected by the inclusion. These are followed by pulses with progressively lower amplitudes, which are the result of reverberations between the flat surface and the inclusion. As these pulses reflect back and forth between these boundaries, they lose energy, and eventually dissipate. The time-of-flight of each of these pulses corresponds to its travel path. The pulse travelling around the inclusion is not easily visible in the time signals because its energy is very small in comparison with the energy of the reflected pulses. The train pulses sequence, in the case of the kidney inclusion, is complicated as it is again the result of the multi-reverberations at the concave part of the inclusion. The amplitude of the responses reveals that the kidney-shaped inclusion allows the energy of the response to be focalized at the top of the inclusion. The circular inclusion, on the other hand, is the one that better dispels the energy. Notice that in this case, the response at receiver $\mathrm{R} 2$ is the biggest, while that at receiver $\mathrm{R} 1$ is the smallest.

\section{Conclusions}

A discrete integration over wavenumbers and frequencies 
has been used to compute the 3D scattered field generated by harmonic spherical wave pressure illuminating cylindrical fixed irregular inclusions in an unbounded and halfspace homogeneous fluid. This technique has been applied to the study of the scattering of waves generated by three different shapes of inclusions, namely circular, oval and kidney. The discretization of the wavenumber-frequency integral transform presented is mathematically equivalent to a periodic sequence of sources, parallel to the axis of the cylinder, that are also periodic in time. We have removed the effects of these periodicities by using complex frequencies.

The main features and the spectral representation of the signals scattered by these inclusions have been described. It has been shown that, although time records may appear complicated, the arrival of various pulses at the receivers can be understood in terms of the ray theory of geometric acoustics. The results obtained are consistent with predictions by ray acoustics, and are used by the authors to elucidate the most important aspect of wave acoustics, that can be used as the basis for the development of non-destructive testing and imaging methods.

It has been shown that the spectra of the response of the inclusions studied exhibit a regular peak and valley structure. The oscillations are due to interference between the waves reflected and diffracted by the boundary of the inclusion and the reflected waves at the flat surface, in the case of the half-space.

\section{Appendix A}

The appendix presents in condensed form the closed form solution to evaluate the $3 \mathrm{D}$ field generated by a point pressure load in the presence of a fixed circular cylindrical cavity of infinite length submerged in a fluid medium.

Since the method used for finding the closed form solution is well known, only a sketch of the formulation is given here [24]. The incident pressure field (Eq. (3)) may be written in terms of waves centred at the axis, which can be achieved using Graf's addition theorem, leading to the expression (in cylindrical coordinates):

$p_{\text {inc }}\left(\omega, r, \theta, \kappa_{z}\right)=-\frac{\mathrm{i} A}{2} \sum_{n=0}^{\infty}(-1)^{n} \varepsilon_{n} H_{n}^{(2)}\left(\kappa_{\alpha} r_{0}\right) J_{n}\left(k_{\alpha} r\right) \cos n \theta$

in which the $J_{n}^{(2)}(\cdots)$ are Bessel functions of the first kind and order $n, \theta$ is the azimuth, and

$\varepsilon_{n}=\left\{\begin{array}{cc}1 / 2 & \text { if } n=0 \\ 1 & \text { if } n \neq 0\end{array}\right.$.

$r=\sqrt{x^{2}+y^{2}}=$ radial distance to the receiver $r_{0}=\sqrt{x_{0}^{2}}=\left|x_{0}\right|=$ radial distance to the source

$\cos \theta=x / r$.

The scattered field can be expressed in a form similar to that of the incident field, namely

$p_{\text {sca }}\left(\omega, r, \theta, k_{z}\right)=\sum_{n=0}^{\infty} A_{n} H_{n}^{(2)}\left(k_{\alpha} r\right) \cos n \theta$

in which the subscript sca denotes the scattered field. The unknown coefficient $A_{n}$ is obtained by imposing a null pressure wave velocity along the normal to the surface of the cylinder at $r=a$.

$A_{n}=\frac{\varepsilon_{n}(-1)^{n} H_{n}^{(2)}\left(\kappa_{\alpha} r_{0}\right)\left[\left(k_{\alpha} a\right) J_{n+1}\left(k_{\alpha} a\right)-n J_{n}\left(k_{\alpha} a\right)\right]}{\left[n H_{n}^{(2)}\left(k_{\alpha} a\right)-\left(k_{\alpha} a\right) H_{n+1}^{(2)}\left(k_{\alpha} a\right)\right]}$.

\section{References}

[1] Trifunac MD. Surface motion of a semi-cylindrical alluvial valley for incident plane SH waves. Bull Seism Soc Am 1971;61:1755-70.

[2] Wong HL, Trifunac MD. Surface motion of semi-elliptical alluvial valley for incident plane SH-waves. Bull Seism Soc Am 1974;64:1389-403.

[3] Lee VW. On deformations near circular underground cavity subjected to incident plane SH waves. Symposium of Applied Computer Methods in Engineering, University of Southern California, Los Angeles, CA, 1977. p. 951-61.

[4] Datta SK, Shah AH. Scattering of SH-waves by embedded cavities. Wave Motion 1982;4:265-83.

[5] Lee VW. Three-dimensional diffraction of elastic waves by a spherical cavity in an elastic half-space. 1: Closed-form solutions. Soil Dynam Earthquake Engng 1988;7:149-61.

[6] Lee VW, Karl JA. Diffraction of SV waves by underground circular, cylindrical cavities. Soil Dynam Earthquake Engng 1992;11:445-56.

[7] Sanchez-Sesma FJ. Diffraction of elastic waves by three dimensional surface irregularities. Bull Seism Soc Am 1983;73:1621-36.

[8] Moeen-Vaziri N, Trifunac MD. Scattering and diffraction of plane P and SV waves by two-dimensional inhomogeneities: Part II. Soil Dynam Earthquake Engng 1988;7:189-200.

[9] Lee VW, Wu X. Application of the weighted residual method to diffraction by 2-D canyons of arbitrary shape: II. Incident P, SV and Rayleigh waves. Soil Dynam Earthquake Engng 1994;13:36575 .

[10] Waas G. Linear two-dimensional analysis of soil dynamics problems in semi-infinite layered media. PhD dissertation, University of California, Berkeley, CA, 1972.

[11] Lysmer J, Udaka T, Seed HB, Hwang R. LUSH-A computer program for complex response analysis of soil-structure systems. Report No. EERC 74-4, Earthquake Engineering Research Center, University of California, Berkeley, CA, 1974.

[12] Kausel E. Forced vibrations of circular foundations in layered media, MIT Research Report 70-3, Department of Civil Engineering, Massachusetts Institute of Technology, Cambridge, MA, 1974.

[13] Ohtsuki A, Harumi K. Effect of topography and subsurface inhomogeneities on seismic SV waves. Earthquake Engng Struct Dynam 1983;11:441-62.

[14] Dawson TW, Fawcett JA. A boundary integral equation method for acoustic scattering in a waveguide with nonplanar surfaces. J Acoust Soc Am 1990;87:1110-25.

[15] Fawcett JA. The computational of the scattered pressure field from a 
cylinder embedded between two half-spaces with different densities. J Acoust Soc Am 1996;99:2435-8.

[16] Fawcett JA. Acoustic scattering from cylindrical objects embedded between two half-spaces. J Acoust Soc Am 1996;100:3053-60.

[17] Lacerda LA, Wrobel LC, Power H, Mansur WJ. A novel boundary integral formulation for three-dimensional analysis of thin acoustic barriers over an impedance plane. J Acoust Soc Am 1998;104:671-8.

[18] Shah AH, Wong KC, Datta SK. Diffraction of plane SH waves in a half-space. Earthquake Engng Struct Dynam 1982;10:519-28.

[19] Lim R. Acoustic scattering by a partially buried three dimensional elastic obstacle. J Acoust Soc Am 1998;104:769-82.
[20] Bouchon M, Aki K. Discrete wave-number representation of seismicsource wave field. Bull Seism Soc Am 1977;67:259-77.

[21] Phinney RA. Theoretical calculation of the spectrum of first arrivals in layered elastic mediums. J Geophys Res 1965;70:5107-23.

[22] Manolis GD, Beskos DE. Boundary element methods in elastodynamics, London: Unwin Hyman/Chapman and Hall, 1988.

[23] Kausel E, Roesset JM. Frequency domain analysis of undamped systems. J Engng Mech ASCE 1992;118(4):721-34.

[24] Tadeu AJB. Modelling and seismic imaging of buried structures. $\mathrm{PhD}$ dissertation, Department of Civil Engineering, Massachusetts Institute of Technology, Cambridge, MA, 1992. 